Article

\title{
Which Out-of-Hospital Cardiac Arrest Patients without ST-Segment Elevation Benefit from Early Coronary Angiography? Results from the Korean Hypothermia Network Prospective Registry
}

Hwan Song ${ }^{1} \mathbb{D}$, Hyo Joon Kim ${ }^{1}$, Kyu Nam Park ${ }^{1}$, Soo Hyun Kim ${ }^{2} \mathbb{D}$, Won Young Kim ${ }^{3} \mathbb{D}$, Byung Kook Lee ${ }^{4} \mathbb{D}$, In Soo Cho ${ }^{5}$, Jae Hoon Lee ${ }^{6}(\mathbb{D})$, Chun Song Youn ${ }^{1, *(\mathbb{D})}$ and on behalf of the Korean Hypothermia Network Investigators ${ }^{\dagger}$

Citation: Song, H.; Kim, H.J.; Park, K.N.; Kim, S.H.; Kim, W.Y.; Lee, B.K.; Cho, I.S.; Lee, J.H.; Youn, C.S.; on behalf of the Korean Hypothermia Network Investigators Which Out-of-Hospital Cardiac Arrest Patients without ST-Segment Elevation Benefit from Early Coronary Angiography? Results from the Korean Hypothermia Network Prospective Registry. J. Clin. Med. 2021, 10, 439. https://doi.org/ $10.3390 /$ jcm10030439

Received: 1 December 2020 Accepted: 20 January 2021 Published: 23 January 2021

Publisher's Note: MDPI stays neutral with regard to jurisdictional claims in published maps and institutional affiliations.

Copyright: (c) 2021 by the authors. Licensee MDPI, Basel, Switzerland. This article is an open access article distributed under the terms and conditions of the Creative Commons Attribution (CC BY) license (https:// creativecommons.org/licenses/by/ $4.0 /)$.
1 Department of Emergency Medicine, Seoul St. Mary Hospital, College of Medicine, The Catholic University of Korea, Seoul 06591, Korea; cmcmdsong@gmail.com (H.S.); liebestest@hanmail.net (H.J.K.); emsky@catholic.ac.kr (K.N.P.)

2 Department of Emergency Medicine, Eunpyeong St. Mary Hospital, College of Medicine, The Catholic University of Korea, Seoul 03312, Korea; unidgirl@catholic.ac.kr

3 Asan Medical Center, Department of Emergency Medicine, Ulsan University College of Medicine, Seoul 05505, Korea; wonpia73@naver.com

4 Department of Emergency Medicine, Chonnam National University Medical School, Gwangju 61469, Korea; bbukkuk@hanmail.net

5 Department of Emergency Medicine, Hanil General Hospital, Korea Electric Power Medical Corporation, Seoul 01450, Korea; mensa@hanmail.net

6 Department of Emergency Medicine, Dong-A University College of Medicine, Busan 49201, Korea; leetoloc@dau.ac.kr

* Correspondence: ycs1005@catholic.ac.kr; Tel.: +82-2-2258-1988; Fax: +82-2-2258-1997

$\dagger$ Membership of Korean Hypothermia Network Investigators is provided in Acknowledgments section.

Abstract: The effect of early coronary angiography (CAG) in out-of-hospital cardiac arrest (OHCA) patients without ST-elevation (STE) is still controversial. It is not known which subgroups of patients without STE are the most likely to benefit. The objective of this study was to evaluate the association between emergency CAG and neurologic outcomes and identify subgroups with improved outcomes when emergency CAG was performed. This prospective, multicenter, observational cohort study was based on data from the Korean Hypothermia Network prospective registry (KORHN-PRO) 1.0. Adult OHCA patients who were treated with targeted temperature management (TTM) without any obvious extracardiac cause were included. Patients were dichotomized into early CAG $(\leq 24 \mathrm{~h})$ and no early CAG ( $>24 \mathrm{~h}$ or not performed) groups. High-risk patients were defined as having the Global Registry of Acute Coronary Events (GRACE) score > 140, time from collapse to return of spontaneous circulation (ROSC) $>30 \mathrm{~min}$, lactate level $>7.0 \mathrm{mmol} / \mathrm{L}$, arterial $\mathrm{pH}<7.2$, cardiac enzyme elevation and ST deviation. The primary outcome was good neurologic outcome at 6 months after OHCA. Of the 1373 patients from the KORHN-PRO 1.0 database, 678 patients met the inclusion criteria. The early CAG group showed better neurologic outcomes at 6 months after cardiac arrest (CA) (adjusted odds ratio: $2.21(1.27-3.87), p=0.005)$. This was maintained even after propensity score matching (adjusted odds ratio: 2.23 (1.39-3.58), $p<0.001)$. In the subgroup analysis, high-risk patients showed a greater benefit from early CAG. In contrast, no significant association was found in low-risk patients. Early CAG was associated with good neurologic outcome at 6 months after CA and should be considered in high-risk patients.

Keywords: out-of-hospital cardiac arrest; cardiopulmonary resuscitation; coronary angiography; outcome 


\section{Introduction}

The survival rate after out-of-hospital cardiac arrest (OHCA) remains low despite recent advances in critical care $[1,2]$. Considering that coronary artery disease is the most common cause of OHCA, early coronary reperfusion may improve outcomes after OHCA [3-5]. Current guidelines highlight the role of emergency coronary angiography (CAG) and percutaneous coronary intervention (PCI) for OHCA patients with ST-segment elevation (STE) on electrocardiogram (ECG) after the return of spontaneous circulation (ROSC) [6,7]. However, the effect of emergency CAG on OHCA patients without STE is still controversial $[6,8,9]$.

Some recent studies have found coronary occlusions in approximately $30 \%$ of patients without STE [10], and early coronary revascularization may increase the survival rate through hemodynamic improvement. Moreover, improvement in hemodynamic status improves heart function and brain blood flow, which can improve neurological outcomes [11]. However, implementing this invasive strategy in routine clinical practice has several logistical and organizational problems. Furthermore, CAG could delay targeted temperature management (TTM), which is standard care after OHCA, and could delay the appropriate diagnosis of other possible causes of cardiac arrest (CA) [11-15]. Observational studies have shown conflicting results regarding the effect of early CAG on the outcome of patients without STE [9,16-19]. In a recent randomized controlled trial (the coronary angiography after cardiac arrest (COACT) trial), immediate angiography was not superior to delayed angiography among patients who had no signs of STE myocardial infarction after ROSC [20]. However, the COACT trial only includes patients with a shockable rhythm, making it difficult to generalize these results to all cardiac arrest patients. Despite limited evidence, recent postcardiac arrest care guidelines recommend early CAG in selected patients after ROSC, even for patients without STE $[7,21,22]$. Surprisingly, no study has investigated the selection of candidates for emergency CAG based on patient data available at the time of hospital admission. Therefore, it is important to identify subgroups in which emergency CAG in patients without STE can lead to improved prognosis.

The purpose of this study was to examine the association between emergency CAG and outcomes in patients without STE after OHCA and to identify subgroups with improved outcomes when emergency CAG was performed.

\section{Methods}

\subsection{Study Design and Setting}

This was a prospective, multicenter, observational cohort study. Data were collected from the Korean Hypothermia Network prospective registry (KORHN-PRO) 1.0 between October 2015 and December 2018. KORHN is a multicenter clinical research consortium for TTM in South Korea. Twenty-two academic hospitals participated in the KORHN-PRO. The study included an informed consent form approved by all participating hospitals, including the institutional review board (IRB) of Seoul St. Mary's Hospital (XC15OIMI0081K), and the study was registered at the International Clinical Trials Registry Platform (NCT02827422). Written informed consent was obtained from all patients' legal surrogates.

\subsection{Population}

The inclusion criteria of KORHN-PRO were as follows: OHCA regardless of etiology of cardiac arrest, age older than 18 years, unconsciousness (Glasgow Coma Scale score $<8$ ) after ROSC and treatment with TTM. The exclusion criteria were as follows: active intracranial bleeding, acute stroke, known limitations in therapy and a do-not-attempt resuscitation order, known prearrest cerebral performance category (CPC) 3 or 4 , known disease making 6-month survival unlikely, and body temperature $<30{ }^{\circ} \mathrm{C}$ on admission.

For the present trial, we excluded patients who had obvious non-cardiac causes (e.g., hanging, asphyxia, drowning, etc.) and STE on the initial ECG from KORHN-PRO. In addition, we included shockable rhythm patients as well as non-shockable rhythm patients in the study, which is different from the COACT trial. 


\subsection{Variables}

The primary outcome was good neurologic outcome at 6 months. The cerebral performance category (CPC) was used to assess neurologic outcome, with a CPC score of 1-2 considered a good outcome and a CPC of 3-5 a poor outcome.

We collected the following demographic and clinical variables information from the registry: age, sex, comorbidities (previous arrest, previous acute myocardial infarction, previous PCI, previous coronary artery bypass grafting (CABG), angina, arrhythmia, chronic heart failure, cerebrovascular accident (CVA) or transient ischemic attack (TIA), hypertension, diabetes, pulmonary disease, chronic kidney disease, liver cirrhosis, malignancy, smoking, alcohol), resuscitation variables (witnessed arrest, bystander cardiopulmonary resuscitation (CPR), initial rhythm, time from collapse to ROSC), and post-ROSC variables (ST deviation, cardiac enzyme elevation, arterial blood gas analysis, lactate level). The Global Registry of Acute Coronary Events (GRACE) risk score was calculated based on the information from the registry.

\subsection{Definitions}

We defined "early CAG" as performed within $24 \mathrm{~h}$ after ROSC [11,18]. "No early CAG" was defined as CAG performed after $24 \mathrm{~h}$ or not performed at all. ECG patterns were classified as follows: normal, ST depression, left bundle branch block (LBBB), right bundle branch block (RBBB), nonspecific ST or T change. ST depression and nonspecific ST or T change were included in ST deviation. The upper limits of cardiac enzyme levels for troponin-I and troponin-T were defined as $3 \mathrm{ng} / \mathrm{dL}$ and $1.4 \mathrm{ng} / \mathrm{dL}$, respectively.

High-risk patients were defined by a GRACE score $>140$, time from collapse to ROSC $>30 \mathrm{~min}$, lactate level $>7.0 \mathrm{mmol} / \mathrm{L}$ and arterial $\mathrm{pH}<7.2$ [22-24].

\subsection{Statistical Methods}

Normality tests (Shapiro-Wilk test) were performed for continuous variables, and continuous variables are presented as the means with the standard deviation (mean $\pm \mathrm{sd}$ ) or as median values with interquartile ranges, as appropriate. Categorical variables are presented as frequencies and percentages. For patient characteristics and comparisons between groups, we used Student's t-test or the Mann-Whitney U test for continuous variables and Fisher's exact test and the chi-square test for categorical variables.

The baseline characteristics of our cohorts are not balanced. Thus, 1:1 or 1:M matching was applied for balanced covariates. By comparison group (early CAG and non-early CAG), we calculated the propensity score (which was calculated using logistic regression). After this analysis, we performed the 1:1 and 1:2 matching by nearest method and checked the standardized difference $(d)$. As a result, we used 1 (early CAG):2 (non-early CAG) matching data for this study.

After propensity score matching, univariate analysis was performed to determine the covariates for neurologic outcome at 6 months after CA. Variables with a $p$-value $\geq 0.05$ on univariate analysis were excluded from the multivariate logistic regression model. To examine the association between early CAG and good neurologic outcome at 6 months after CA, multivariate logistic regression analyses with backward elimination were performed. We then performed multivariate logistic regression analysis among each subgroup of high-risk patients (GRACE score > 140, time from collapse to ROSC > $30 \mathrm{~min}$, lactate level $>7.0 \mathrm{mmol} / \mathrm{L}$ and arterial $\mathrm{pH}<7.2)$ and low-risk patients. The association between early CAG and outcome was quantified using odds ratios (ORs) with $95 \%$ confidence intervals (CIs).

Statistical analysis was performed using SAS version 9.4 (SAS Institute, Inc., Cary, NC, USA) and R version 4.0.3, and $p$-values $\leq 0.05$ were considered statistically significant. 


\section{Results}

\subsection{Study Population}

During the study period, a total of 1373 OHCA patients older than 18 years who were treated with TTM were enrolled in KORHN-PRO. Among them, 521 patients were excluded due to obvious non-cardiac causes of arrest, and 11 patients were excluded due to lack of ECG data. STE was found in 163 patients, and the remaining 678 patients were ultimately included in this study (Figure 1). CAG was performed within $24 \mathrm{~h}$ in 231 patients (early CAG group), and the remaining 447 patients either underwent $C A G$ after $24 \mathrm{~h}$ or were not treated with CAG (no early CAG group).

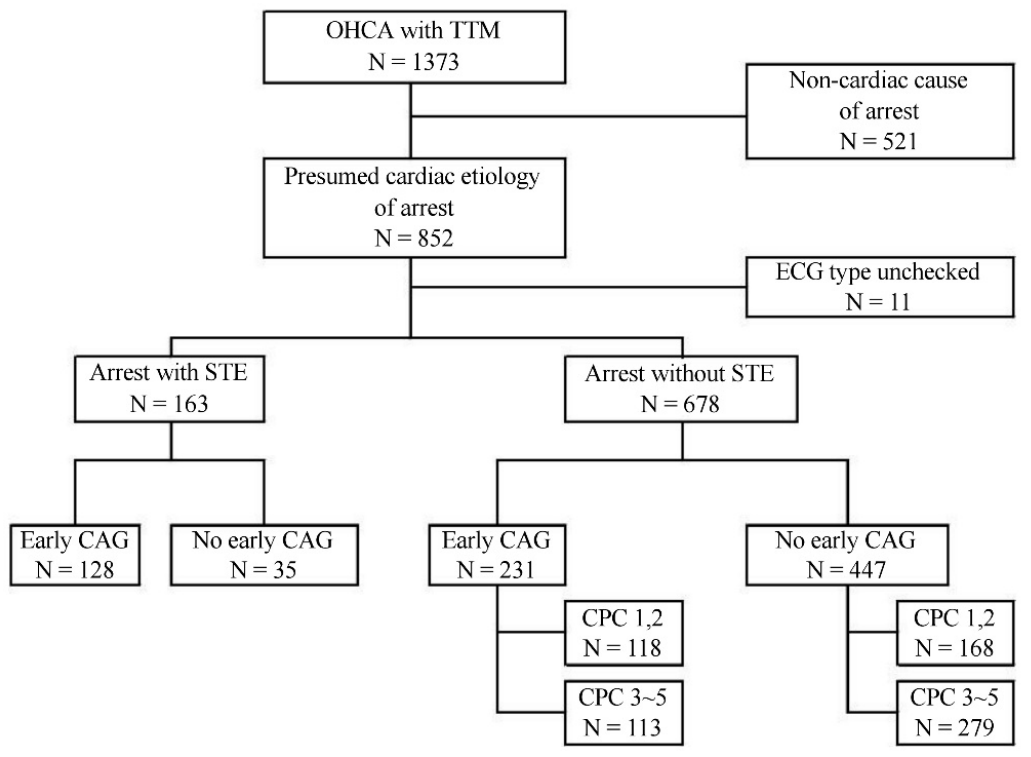

Figure 1. Flow chart of patients. Abbreviations: OHCA = out-of-hospital cardiac arrest, TTM = targeted temperature management, ECG = electrocardiogram, STE = ST segment elevation, $\mathrm{CAG}=$ coronary angiography, $\mathrm{CPC}=$ cerebral performance category.

The mean age was not significantly different between the early CAG group (58.5 \pm 13.8$)$ and the no early CAG group $(59.6 \pm 16.1, p=0.357)$, and $497(73.3 \%)$ patients were male. There were no significant differences between the early CAG group and the no early CAG group in comorbidities, except chronic heart failure and pulmonary disease. The proportion of good neurologic outcome at 6 months after CA was higher in the early CAG group than in the no early CAG group $(51.1 \%$ vs. $37.6 \%, p<0.001)$.

An initial shockable rhythm was more common in the early CAG group than in the no early CAG group $(68.3 \%$ vs. $47.9 \%, p<0.001)$. Moreover, cardiac enzyme elevation was also more common in the early CAG group than in the no early CAG group $(83.9 \%$ vs. $72.0 \%, p<0.001)$. The time from collapse to ROSC and GRACE score showed no significant differences between the two groups (Table 1).

Table 1. Baseline characteristics of patients with out-of-hospital cardiac arrest without ST-segment elevation.

\begin{tabular}{|c|c|c|c|c|c|c|}
\hline & \multicolumn{3}{|c|}{ Before Propensity Score Matching } & \multicolumn{3}{|c|}{ After Propensity Score Matching } \\
\hline & $\begin{array}{c}\text { No Early CAG } \\
(\mathrm{N}=447)\end{array}$ & $\begin{array}{l}\text { Early CAG } \\
(\mathrm{N}=231)\end{array}$ & $p$ Value & $\begin{array}{c}\text { No Early CAG } \\
(\mathrm{N}=364)\end{array}$ & $\begin{array}{c}\text { Early CAG } \\
(\mathrm{N}=182)\end{array}$ & $p$ Value \\
\hline Age, years & $59.61 \pm 16.08$ & $58.52 \pm 13.75$ & 0.357 & $58.76 \pm 15.90$ & $58.26 \pm 14.05$ & 0.399 \\
\hline Sex, male & $317(70.9)$ & $180(77.9)$ & 0.051 & $262(72.0)$ & $139(76.4)$ & 0.320 \\
\hline Comorbidities & & & & & & \\
\hline Previous arrest & $10(2.2)$ & $2(0.9)$ & 0.237 & $7(1.9)$ & $2(1.1)$ & 0.725 \\
\hline Previous AMI & $31(6.9)$ & $24(10.4)$ & 0.118 & $24(6.6)$ & $16(8.8)$ & 0.450 \\
\hline Previous PCI & $18(4.0)$ & $15(6.5)$ & 0.157 & $16(4.4)$ & $12(6.6)$ & 0.373 \\
\hline
\end{tabular}


Table 1. Cont.

\begin{tabular}{|c|c|c|c|c|c|c|}
\hline & \multicolumn{3}{|c|}{ Before Propensity Score Matching } & \multicolumn{3}{|c|}{ After Propensity Score Matching } \\
\hline & $\begin{array}{l}\text { No Early CAG } \\
\quad(\mathrm{N}=447)\end{array}$ & $\begin{array}{l}\text { Early CAG } \\
(N=231)\end{array}$ & $p$ Value & $\begin{array}{l}\text { No Early CAG } \\
\quad(\mathrm{N}=364)\end{array}$ & $\begin{array}{l}\text { Early CAG } \\
(\mathrm{N}=182)\end{array}$ & $p$ Value \\
\hline Previous CABG & $6(1.3)$ & $5(2.2)$ & 0.523 & $4(1.1)$ & $3(1.6)$ & 0.691 \\
\hline Angina & $31(6.9)$ & $24(10.4)$ & 0.118 & $21(5.8)$ & $15(8.2)$ & 0.360 \\
\hline Arrhythmia & $32(7.2)$ & $15(6.5)$ & 0.747 & $29(8.0)$ & $14(7.7)$ & 1.000 \\
\hline Chronic heart failure & $28(6.3)$ & $6(2.6)$ & 0.038 & $19(5.2)$ & $6(3.3)$ & 0.426 \\
\hline CVA or TIA & $27(6.0)$ & $9(3.9)$ & 0.238 & $15(4.1)$ & $5(2.7)$ & 0.573 \\
\hline Hypertension & $189(42.3)$ & $86(37.2)$ & 0.204 & $145(39.8)$ & $71(39.0)$ & 0.926 \\
\hline Diabetes mellitus & $118(26.4)$ & $56(24.2)$ & 0.543 & $90(24.7)$ & $42(23.1)$ & 0.750 \\
\hline Pulmonary disease & $30(6.7)$ & $2(0.9)$ & 0.001 & $14(3.8)$ & $2(1.1)$ & 0.127 \\
\hline Chronic kidney disease & $41(9.2)$ & $12(5.2)$ & 0.068 & $23(6.3)$ & $8(4.4)$ & 0.472 \\
\hline Liver cirrhosis & $7(1.6)$ & $0(0.0)$ & 0.102 & $0(0.0)$ & $0(0.0)$ & 1.000 \\
\hline Malignancy & $27(6.0)$ & $10(4.3)$ & 0.353 & $19(5.2)$ & $9(4.9)$ & 1.000 \\
\hline Smoking & $111(25.5)$ & $80(36.0)$ & 0.005 & $62(22.6)$ & $49(32.7)$ & 0.025 \\
\hline Alcohol & $144(33.0)$ & $92(41.4)$ & 0.033 & $91(33.2)$ & $64(42.7)$ & 0.053 \\
\hline \multicolumn{7}{|l|}{ Resuscitation variables } \\
\hline Witness & $354(79.6)$ & $184(80.0)$ & 0.891 & $292(80.2)$ & $144(79.1)$ & 0.850 \\
\hline Bystander & $281(63.6)$ & $154(67.5)$ & 0.308 & $235(64.6)$ & $127(69.8)$ & 0.263 \\
\hline Shockable & $201(47.9)$ & $138(68.3)$ & $<0.001$ & $186(51.1)$ & $125(68.7)$ & $<0.001$ \\
\hline $\begin{array}{l}\text { Time from collapse to ROSC, } \\
\text { min }\end{array}$ & $31.77 \pm 22.38$ & $32.08 \pm 21.37$ & 0.862 & $31.65 \pm 24.08$ & $32.72 \pm 20.59$ & 0.248 \\
\hline $\begin{array}{l}\text { Time from collapse to ROSC > } \\
\qquad 30 \mathrm{~min}\end{array}$ & $200(44.7)$ & $100(43.3)$ & 0.718 & $158(43.4)$ & $83(45.6)$ & 0.692 \\
\hline \multicolumn{7}{|l|}{ Post-resuscitation variables } \\
\hline ST deviation & $303(67.8)$ & $167(72.3)$ & 0.228 & 247 (67.9) & $129(70.9)$ & 0.535 \\
\hline Cardiac enzyme elevation & $321(72.0)$ & $193(83.9)$ & 0.001 & $263(72.3)$ & $150(82.4)$ & 0.012 \\
\hline Arterial $\mathrm{pH}$ & $7.09 \pm 0.21$ & $7.13 \pm 0.18$ & 0.023 & $7.10 \pm 0.21$ & $7.13 \pm 0.18$ & 0.117 \\
\hline Arterial $\mathrm{pH}<7.2$ & $277(63.8)$ & $126(57.5)$ & 0.118 & $223(61.3)$ & $104(57.1)$ & 0.405 \\
\hline Lactate, mmol/L & $9.09 \pm 4.92$ & $9.15 \pm 4.85$ & 0.884 & $8.99 \pm 5.07$ & $9.12 \pm 5.04$ & 0.885 \\
\hline Lactate $>7 \mathrm{mmol} / \mathrm{L}$ & $267(62.1)$ & $142(64.3)$ & 0.589 & $228(62.6)$ & $117(64.3)$ & 0.778 \\
\hline GRACE score & $179.85 \pm 52.74$ & $178.23 \pm 48.58$ & 0.696 & $177.45 \pm 54.51$ & $174.74 \pm 47.97$ & 0.524 \\
\hline $\begin{array}{c}\text { GRACE score }>140 \\
\text { TTM variables }\end{array}$ & $337(75.4)$ & $183(79.2)$ & 0.264 & $269(73.9)$ & $143(78.6)$ & 0.276 \\
\hline Target temperature of $33^{\circ} \mathrm{C}$ & $381(85.2)$ & $168(72.7)$ & $<0.001$ & $251(89.6)$ & $119(76.3)$ & $<0.001$ \\
\hline Target temperature of $36^{\circ} \mathrm{C}$ & $66(14.8)$ & $63(27.3)$ & $<0.001$ & $29(10.4)$ & $37(23.7)$ & $<0.001$ \\
\hline $\begin{array}{l}\text { Time to achieve target } \\
\text { temperature, } \text { min }\end{array}$ & $424.60 \pm 277.20$ & $484.90 \pm 290.87$ & 0.009 & $389.47 \pm 239.36$ & $490.44 \pm 313.89$ & $<0.001$ \\
\hline Survival discharge & $255(57.0)$ & $163(70.6)$ & 0.001 & $164(58.6)$ & $112(71.8)$ & 0.006 \\
\hline Survival at 6 months & $207(47.4)$ & $131(58.2)$ & 0.008 & $131(47.3)$ & $93(60.4)$ & 0.009 \\
\hline $\begin{array}{l}\text { Good neurologic outcome at } \\
6 \text { months }\end{array}$ & $168(37.6 \%)$ & $118(51.1 \%)$ & 0.001 & $107(38.6)$ & $82(53.3)$ & 0.003 \\
\hline
\end{tabular}

$p<0.05$ are presented in bold. Abbreviations: $\mathrm{AMI}=$ acute myocardial infarction, $\mathrm{CVA}=$ cerebrovascular accident, TIA $=$ transient ischemic attack, HBP = hypertension, DM = diabetes mellitus, ABGA = arterial blood gas analysis, GRACE = Global Registry of Acute Coronary Events, ROSC: return of spontaneous circulation, PCI: percutaneous coronary intervention, CABG: coronary artery bypass grafting, TTM: targeted temperature management.

\subsection{ECG and CAG Findings}

ST depression was more common in the early CAG group (102 (44.2\%) vs. $106(23.7 \%)$, $p<0.001)$, while nonspecific ST or T was more common in the no early CAG group (212 $(47.4 \%)$ vs. $67(29.0 \%), p<0.001)$.

The median time from ROSC to CAG was $2.2 \mathrm{~h}$ (interquartile range (IQR) 1.5-3.3 h) in the early CAG group and $176.7 \mathrm{~h}$ (IQR 12.6-229.5) in the no early CAG group. Coronary artery disease, defined as more than $50 \%$ stenosis of any coronary artery, was $41.1 \%$ in the early CAG group, and the left anterior descending artery was the most commonly involved artery $(66.3 \%)$. Notably, CAG was performed in $104(23.3 \%)$ patients in the no early CAG group, and only $26(5.8 \%)$ patients required subsequent PCI. In contrast, $76(32.9 \%)$ patients in the early CAG group underwent PCI (Table 2). 
Table 2. Coronary angiography and electrocardiogram (ECG) findings.

\begin{tabular}{|c|c|c|c|}
\hline & $\begin{array}{l}\text { No Early CAG } \\
\quad(\mathrm{N}=447)\end{array}$ & $\begin{array}{c}\text { Early CAG } \\
(N=231)\end{array}$ & $p$ Value \\
\hline $\begin{array}{l}\text { Time to CAG, hours, median } \\
\text { (IQR) }\end{array}$ & $176.66(125.68,229.52)$ & $2.2(1.54,3.33)$ & $<0.001$ \\
\hline No CAG & $343(76.7 \%)$ & $\mathrm{N} / \mathrm{A}$ & \\
\hline $\mathrm{CAD}(>=50 \%)$ & $32(7.2 \%)$ & $95(41.1 \%)$ & $<0.001$ \\
\hline 1VD & $18 / 32(56.3 \%)$ & $53 / 95(55.8 \%)$ & 0.964 \\
\hline $2 \mathrm{VD}$ & $8 / 32(25.0 \%)$ & $19 / 95(20.0 \%)$ & 0.550 \\
\hline $3 \mathrm{VD}$ & $6 / 32(18.8 \%)$ & $21 / 95(22.1 \%)$ & 0.688 \\
\hline LAD & $23 / 32(71.9 \%)$ & $63 / 95(66.3 \%)$ & 0.561 \\
\hline $\mathrm{LCx}$ & $12 / 32(37.5 \%)$ & $49 / 95(51.6 \%)$ & 0.168 \\
\hline RCA & $17 / 32(53.1 \%)$ & $42 / 95(44.2 \%)$ & 0.382 \\
\hline Left main & $0 / 32(0 \%)$ & $7 / 95(7.4 \%)$ & 0.190 \\
\hline Coronary intervention & $26(5.8 \%)$ & $76(32.9 \%)$ & $<0.001$ \\
\hline ST depression & $106(23.7 \%)$ & $102(44.2 \%)$ & $<0.001$ \\
\hline LBBB & $18(4.0 \%)$ & $10(4.36 \%)$ & 0.851 \\
\hline RBBB & $101(22.6 \%)$ & $50(21.6 \%)$ & 0.778 \\
\hline Non-specific ST or T & $212(47.4 \%)$ & $67(29.0 \%)$ & $<0.001$ \\
\hline Normal ST segment and T & $76(17.0 \%)$ & $27(11.7 \%)$ & 0.068 \\
\hline
\end{tabular}

\subsection{Association between Early CAG and Outcomes in the Whole Population}

In the univariate analysis, age, previous PCI, history of high blood pressure (HBP), diabetes mellitus (DM), pulmonary disease and chronic kidney disease, witnessed arrest, bystander CPR, initial shockable rhythm, time from collapse to ROSC $>30 \mathrm{~min}$, arterial $\mathrm{pH}$ $<7.2$, lactate $>7.0 \mathrm{mmol} / \mathrm{L}$ and GRACE score $>140$ were associated with good neurologic outcome at 6 months after CA.

In the multivariate logistic regression analysis, the early CAG group was associated with good neurologic outcome at 6 months after CA (adjusted OR: $2.21(1.27-3.87), p=0.005$ ) (Table 3). The cardiovascular Sequential Organ Failure Assessment (SOFA) score from the third to seventh day after ROSC differed between the early CAG group and the no early CAG group in patients with a GRACE score of 140 or higher immediately after ROSC. However, in the group with a GRACE score $\leq 140$, the cardiovascular SOFA score showed no significant difference between the two groups (Figure 2).

After propensity score matching, early CAG was associated with good neurologic outcome at 6 months after CA (adjusted OR: 2.23 (1.39-3.58), $p<0.001$ ) (Table 3).

Table 3. Association between 6-month good neurologic outcome and baseline variables.

\begin{tabular}{|c|c|c|c|c|c|c|c|c|}
\hline & \multicolumn{4}{|c|}{ Before Propensity Score Matching } & \multicolumn{4}{|c|}{ After Propensity Score Matching } \\
\hline & \multicolumn{2}{|c|}{ Crude } & \multicolumn{2}{|c|}{ Adjusted } & \multicolumn{2}{|c|}{ Crude } & \multicolumn{2}{|c|}{ Adjusted } \\
\hline & OR $(95 \% \mathrm{CI})$ & $p$ Value & OR $(95 \% \mathrm{CI})$ & $p$ Value & OR $(95 \% \mathrm{CI})$ & $p$ Value & OR $(95 \% \mathrm{CI})$ & $p$ Value \\
\hline Early CAG & $\begin{array}{c}1.73 \\
(1.26-2.39)\end{array}$ & 0.001 & $\begin{array}{c}2.21 \\
(1.27-3.87)\end{array}$ & 0.005 & $\begin{array}{c}1.83 \\
(1.25-2.67)\end{array}$ & 0.002 & $\begin{array}{c}2.23 \\
(1.39-3.58)\end{array}$ & $<0.001$ \\
\hline Age, years & $\begin{array}{c}0.95 \\
(0.94-0.96)\end{array}$ & $<0.001$ & & & $\begin{array}{c}0.95 \\
(0.94-0.96)\end{array}$ & $<0.001$ & $\begin{array}{c}0.98 \\
(0.96-1.00)\end{array}$ & 0.011 \\
\hline Sex (male) & $\begin{array}{c}0.72 \\
(0.51-1.03)\end{array}$ & 0.069 & & & $\begin{array}{c}1.35 \\
(0.89-2.02)\end{array}$ & 0.155 & & \\
\hline GRACE score $(>140)$ & $\begin{array}{c}0.08 \\
(0.05-0.13)\end{array}$ & $<0.001$ & $\begin{array}{c}0.15 \\
(0.07-0.30)\end{array}$ & $<0.001$ & $\begin{array}{c}0.08 \\
(0.05-0.13)\end{array}$ & $<0.001$ & $\begin{array}{c}0.24 \\
(0.13-0.47)\end{array}$ & $<0.001$ \\
\hline \multicolumn{9}{|l|}{ Comorbidities } \\
\hline Previous arrest & $\begin{array}{c}1.02 \\
(0.32-3.25)\end{array}$ & 0.971 & & & $\begin{array}{c}0.61 \\
(0.16-2.25)\end{array}$ & 0.454 & & \\
\hline Previous AMI & $\begin{array}{c}1.55 \\
(0.86-2.79)\end{array}$ & 0.141 & & & $\begin{array}{c}0.48 \\
(0.24-0.96)\end{array}$ & 0.037 & $\begin{array}{c}1.16 \\
(0.52-2.57)\end{array}$ & 0.716 \\
\hline
\end{tabular}


Table 3. Cont.

\begin{tabular}{|c|c|c|c|c|c|c|c|c|}
\hline & \multicolumn{4}{|c|}{ Before Propensity Score Matching } & \multicolumn{4}{|c|}{ After Propensity Score Matching } \\
\hline & \multicolumn{2}{|c|}{ Crude } & \multicolumn{2}{|c|}{ Adjusted } & \multicolumn{2}{|c|}{ Crude } & \multicolumn{2}{|c|}{ Adjusted } \\
\hline & OR $(95 \%$ CI) & $p$ Value & OR $(95 \% \mathrm{CI})$ & $p$ Value & OR $(95 \% \mathrm{CI})$ & $p$ Value & OR $(95 \%$ CI $)$ & $p$ Value \\
\hline Previous PCI & $\begin{array}{c}2.83 \\
(1.21-6.62)\end{array}$ & 0.016 & $\begin{array}{c}0.76 \\
(0.21-2.78)\end{array}$ & 0.675 & $\begin{array}{c}0.42 \\
(0.17-1.05)\end{array}$ & 0.064 & & \\
\hline Previous CABG & $\begin{array}{c}1.96 \\
(0.52-7.47)\end{array}$ & 0.322 & & & $\begin{array}{c}0.34 \\
(0.07-1.78)\end{array}$ & 0.202 & & \\
\hline Angina & $\begin{array}{c}0.80 \\
(0.46-1.39)\end{array}$ & 0.426 & & & $\begin{array}{c}1.10 \\
(0.55-2.17)\end{array}$ & 0.795 & & \\
\hline Arrhythmia & $\begin{array}{c}1.08 \\
(0.59-1.98)\end{array}$ & 0.802 & & & $\begin{array}{c}0.67 \\
(0.34-1.32)\end{array}$ & 0.249 & & \\
\hline Chronic heart failure & $\begin{array}{c}1.80 \\
(0.85-3.83)\end{array}$ & 0.127 & & & $\begin{array}{c}0.42 \\
(0.17-1.04)\end{array}$ & 0.061 & & \\
\hline CVA or TIA & $\begin{array}{c}1.70 \\
(0.82-3.52)\end{array}$ & 0.151 & & & $\begin{array}{c}0.87 \\
(0.32-2.33)\end{array}$ & 0.777 & & \\
\hline HBP & $\begin{array}{c}2.29 \\
(1.66-3.15)\end{array}$ & $<0.001$ & $\begin{array}{c}0.78 \\
(0.44-1.36)\end{array}$ & 0.376 & $\begin{array}{c}0.47 \\
(0.32-0.68)\end{array}$ & $<0.001$ & $\begin{array}{c}0.62 \\
(0.39-1.00)\end{array}$ & 0.052 \\
\hline $\mathrm{DM}$ & $\begin{array}{c}3.33 \\
(2.24-4.95)\end{array}$ & $<0.001$ & $\begin{array}{c}0.57 \\
(0.28-1.15)\end{array}$ & 0.115 & $\begin{array}{c}0.34 \\
(0.21-0.54)\end{array}$ & $<0.001$ & $\begin{array}{c}0.77 \\
(0.44-1.37)\end{array}$ & 0.376 \\
\hline Pulmonary disease & $\begin{array}{c}2.72 \\
(1.16-6.37)\end{array}$ & 0.022 & $\begin{array}{c}2.12 \\
(0.64-7.04)\end{array}$ & 0.222 & $\begin{array}{c}0.30 \\
(0.06-1.47)\end{array}$ & 0.137 & & \\
\hline Chronic kidney disease & $\begin{array}{c}3.00 \\
(1.52-5.94)\end{array}$ & 0.002 & $\begin{array}{c}0.31 \\
(0.09-1.03)\end{array}$ & 0.056 & $\begin{array}{c}0.32 \\
(0.13-0.84)\end{array}$ & 0.020 & $\begin{array}{c}0.30 \\
(0.11-0.84)\end{array}$ & 0.021 \\
\hline malignancy & $\begin{array}{c}1.07 \\
(0.55-2.11)\end{array}$ & 0.836 & & & $\begin{array}{c}1.28 \\
(0.58-2.84)\end{array}$ & 0.548 & & \\
\hline Smoking & $\begin{array}{c}1.28 \\
(0.92-1.80)\end{array}$ & 0.145 & & & $\begin{array}{c}1.00 \\
(0.66-1.52)\end{array}$ & 0.985 & & \\
\hline Alcohol & $\begin{array}{c}1.97 \\
(1.43-2.71)\end{array}$ & $<0.001$ & $\begin{array}{c}1.16 \\
(0.67-2.00)\end{array}$ & 0.597 & $\begin{array}{c}1.57 \\
(1.08-2.30)\end{array}$ & 0.020 & $\begin{array}{c}0.86 \\
(0.54-1.37)\end{array}$ & 0.535 \\
\hline Resuscitation variables & & & & & & & & \\
\hline Witness & $\begin{array}{c}2.53 \\
(1.66-3.85)\end{array}$ & $<0.001$ & $\begin{array}{c}1.87 \\
(0.93-3.79)\end{array}$ & 0.080 & $\begin{array}{c}2.87 \\
(1.71-4.80)\end{array}$ & $<0.001$ & $\begin{array}{c}1.74 \\
(0.98-3.07)\end{array}$ & 0.059 \\
\hline Bystander CPR & $\begin{array}{c}1.42 \\
(1.03-1.97)\end{array}$ & 0.035 & $\begin{array}{c}1.11 \\
(0.63-1.96)\end{array}$ & 0.716 & $\begin{array}{c}1.46 \\
(0.99-2.13)\end{array}$ & 0.055 & & \\
\hline Shockable rhythm & $\begin{array}{c}10.73 \\
(7.23-15.92)\end{array}$ & $<0.001$ & $\begin{array}{c}6.67 \\
(3.76-11.82)\end{array}$ & $<0.001$ & $\begin{array}{c}9.88 \\
(6.41-15.22)\end{array}$ & $<0.001$ & $\begin{array}{c}3.94 \\
(2.47-6.29)\end{array}$ & $<0.001$ \\
\hline ROSC $>30 \mathrm{~min}$ & $\begin{array}{c}0.11 \\
(0.08-0.16)\end{array}$ & $<0.001$ & $\begin{array}{c}0.16 \\
(0.09-0.28)\end{array}$ & $<0.001$ & $\begin{array}{c}0.21 \\
(0.08-0.18)\end{array}$ & $<0.001$ & $\begin{array}{c}0.18 \\
(0.11-0.27)\end{array}$ & $<0.001$ \\
\hline \multicolumn{9}{|c|}{ Post-resuscitation variables } \\
\hline ST deviation & $\begin{array}{c}0.77 \\
(0.55-1.07)\end{array}$ & 0.119 & & & $\begin{array}{c}0.89 \\
(0.60-1.32)\end{array}$ & 0.564 & & \\
\hline Cardiac enzyme elevation & $\begin{array}{c}0.81 \\
(0.57-1.15)\end{array}$ & 0.239 & & & $\begin{array}{c}0.71 \\
(0.47-1.07)\end{array}$ & 0.097 & & \\
\hline Arterial $\mathrm{pH}<7.2$ & $\begin{array}{c}0.20 \\
(0.14-0.28)\end{array}$ & $<0.001$ & $\begin{array}{c}0.48 \\
(0.27-0.85)\end{array}$ & 0.012 & $\begin{array}{c}0.21 \\
(0.14-0.31)\end{array}$ & $<0.001$ & $\begin{array}{c}0.44 \\
(0.27-0.71)\end{array}$ & 0.001 \\
\hline $\begin{array}{c}\text { Lactate }>7.0 \mathrm{mmol} / \mathrm{L} \\
\text { TTM variables }\end{array}$ & $\begin{array}{c}0.31 \\
(0.23-0.44)\end{array}$ & $<0.001$ & $\begin{array}{c}0.70 \\
(0.39-1.24)\end{array}$ & 0.221 & $\begin{array}{c}0.36 \\
(0.25-0.53)\end{array}$ & $<0.001$ & $\begin{array}{c}0.72 \\
(0.44-1.18)\end{array}$ & 0.191 \\
\hline Target temperature of $33^{\circ} \mathrm{C}$ & $\begin{array}{c}1.08 \\
(0.73-1.60)\end{array}$ & 0.701 & & & $\begin{array}{c}0.94 \\
(0.57-1.54)\end{array}$ & 0.793 & & \\
\hline Time to achieve TTM & $\begin{array}{c}0.99 \\
(0.99-1.00)\end{array}$ & $<0.001$ & $\begin{array}{c}1.00 \\
(1.00-1.00)\end{array}$ & 0.654 & $\begin{array}{c}1.00 \\
(1.00-1.00)\end{array}$ & $<0.001$ & $\begin{array}{c}0.76 \\
(0.43-1.36)\end{array}$ & 0.362 \\
\hline
\end{tabular}

$p<0.05$ are presented in bold. Adjusted for CAG, age, GRACE score ( $>140)$, previous PCI, HBP, DM, pulmonary disease, CKD, alcohol, witness, bystander cardiopulmonary resuscitation (CPR), shockable rhythm, time to $\mathrm{ROSC}(>30), \mathrm{ABGA}$ pH $(<7.2)$, lactate $(>7.0)$ and time to achieve TTM. 
(A)

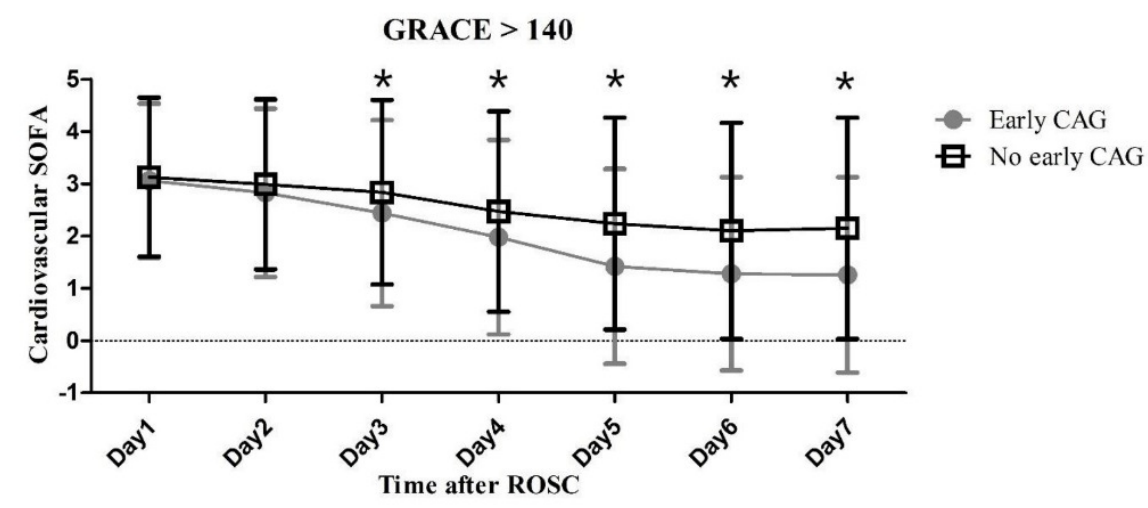

(B)

GRACE $\leq 140$

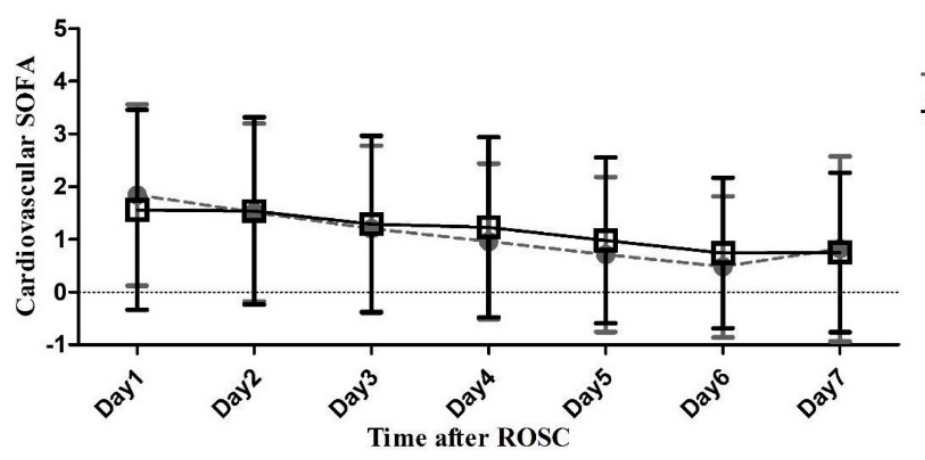

Early CAG

廿 No early CAG

Figure 2. Cardiovascular SOFA score within 7 days after ROSC according to GRACE score. (A) Cardiovascular SOFA score in patients with GRACE score $>140$ (B) Cardiovascular SOFA score in patients with GRACE score $\leq 140 .{ }^{*}$ Indicates a $p$ value of less than 0.05 .

\subsection{Impact of Early CAG in Selected High-Risk Patients}

We performed subgroup analysis between the early CAG group and the no early CAG group to assess good neurologic outcomes at 6 months. Early CAG was associated with good neurologic outcome at 6 months after CA in high-risk patients (defined as GRACE score $>140$, time from collapse to ROSC $>30 \mathrm{~min}$, arterial $\mathrm{pH}<7.2$, lactate $>7.0 \mathrm{mmol} / \mathrm{L}$, cardiac enzyme elevation, and ST deviation). In contrast, no significant association was found among patients with GRACE score $\leq 140$, time from collapse to ROSC $\leq 30 \mathrm{~min}$, lactate level $\leq 7.0 \mathrm{mmol} / \mathrm{L}$, arterial $\mathrm{pH} \geq 7.2$, no cardiac enzyme elevation and no ST deviation (Table 4).

Table 4. The adjusted odds ratios of early CAG for predicting 6-month good neurologic outcome after out-of-hospital cardiac arrest.

\begin{tabular}{ccccc}
\hline & Total Patients & Early CAG & Good Outcome & OR (95\% CI) \\
\hline GRACE score $\leq 140$ & 151 & 45 & 40 & $1.64(0.57-4.72)$ \\
GRACE score $>140$ & 527 & 186 & 78 & $\mathbf{2 . 3 6 ( 1 . 6 1 - 3 . 4 6 )}$ \\
Time from collapse to ROSC $\leq 30 \mathrm{~min}$ & 378 & 131 & 89 & $1.39(0.89-2.18)$ \\
Time from collapse to ROSC $>$ 30 min & 300 & 100 & 29 & $\mathbf{3 . 8 9 ( 2 . 0 5 - 7 . 3 8 )}$ \\
Arterial $\mathrm{pH} \geq 7.2$ & 250 & 93 & 64 & $1.29(0.75-2.23)$ \\
Arterial $\mathrm{pH}<7.2$ & 403 & 126 & 47 & $\mathbf{2 . 0 6}(\mathbf{1 . 3 0 - 3 . 2 6 )}$ \\
\hline
\end{tabular}


Table 4. Cont.

\begin{tabular}{|c|c|c|c|c|}
\hline & Total Patients & Early CAG & Good Outcome & OR $(95 \%$ CI $)$ \\
\hline Lactate $\leq 7.0 \mathrm{mmol} / \mathrm{L}$ & 242 & 79 & 53 & $1.61(0.92-2.83)$ \\
\hline Lactate $>7.0 \mathrm{mmol} / \mathrm{L}$ & 409 & 142 & 60 & $2.10(1.36-3.23)$ \\
\hline ST deviation $(+)$ & 470 & 167 & 84 & $1.91(1.30-2.80)$ \\
\hline ST deviation $(-)$ & 208 & 64 & 34 & $1.46(0.81-2.63)$ \\
\hline Cardiac enzyme elevation $(+)$ & 514 & 193 & 100 & $2.03(1.41-2.93)$ \\
\hline Cardiac enzyme elevation $(-)$ & 162 & 37 & 18 & $1.13(0.54-2.36)$ \\
\hline
\end{tabular}

$p<0.05$ are presented in bold. OR: odds ratio, CI: confidence interval. Adjusted for CAG, age, GRACE score ( $>140)$, previous PCI, HBP, $\mathrm{DM}$, pulmonary disease, CKD, alcohol, witness, bystander CPR, shockable rhythm, time to ROSC $(>30)$, ABGA pH $(<7.2)$, lactate $(>7.0)$ and time to achieve TTM.

\section{Discussion}

The main findings of this study are as follows: first, early CAG was associated with improved long-term neurologic outcome at 6 months after CA in patients treated with TTM. Second, we demonstrated an association between early CAG and improved long-term neurologic outcome among high-risk patients (i.e., GRACE score $>140$, time from collapse to $\mathrm{ROSC}>30 \mathrm{~min}$, lactate level $>7.0 \mathrm{mmol} / \mathrm{L}$, arterial $\mathrm{pH}<7.2$, cardiac enzyme elevation and ST deviation). In contrast, no significant association was found among patients with GRACE score $\leq 140$, time from collapse to ROSC $\leq 30 \mathrm{~min}$, lactate level $\leq 7.0 \mathrm{mmol} / \mathrm{L}$, arterial $\mathrm{pH} \geq 7.2$, no cardiac enzyme elevation and no ST deviation.

Early CAG has the advantage of early implementation of PCI, which can most effectively improve the hemodynamic status of patients with coronary artery disease (CAD), ameliorating the cerebral perfusion of patients and resulting in an improvement in longterm neurologic outcomes. We demonstrated that the cardiovascular SOFA score from the third to the seventh day after ROSC differed between the early CAG group and the no early CAG group in patients with a GRACE score of 140 or higher immediately after ROSC. However, there is no reason for emergency CAG in patients whose neurological outcome is expected to be futile. If there is no STE on the electrocardiogram after CA, there are two important issues when deciding whether to perform emergency CAG: right patients and right timing.

The optimal timing of CAG after CA is still unknown. Kim et al. stated that immediate CAG within $2 \mathrm{~h}$ after ROSC or emergency department presentation had no clear neurological benefit compared with early CAG between $2 \mathrm{~h}$ and $24 \mathrm{~h}$ [3]. In addition, several retrospective studies have defined early CAG as being performed within $24 \mathrm{~h}$ after ROSC $[3,11,25]$. Based on this, we classified patients who underwent CAG within $24 \mathrm{~h}$ as the early CAG group [23]. However, we did not find any evidence for correct timing in this study. Future studies are needed.

According to previous systematic reviews, CAD was diagnosed in one-third of patients who underwent CAG without STE $[10,26]$. In our study, the early CAG group was diagnosed with CAD in $41.1 \%$ of patients, similar to previous systematic reviews. However, in the no early CAG group, CAD was diagnosed in $7.2 \%$ of the patients, and among the no early CAG group, CAD was diagnosed in $30.8 \%$ of the patients who underwent CAG; thus, $C A D$ was diagnosed among patients who underwent CAG at a rate similar to that of previous studies.

Comatose patients after CA with evidence of STE should undergo emergency CAG. However, it is challenging to decide which patients should undergo emergency CAG without evidence of STE, i.e., the "right" patients. Therefore, we defined high-risk patients based on the American Heart Association/American College of Cardiology (AHA/ACC) guidelines and the algorithm proposed by Rab et al. [22,23]. For non-cardiac arrest patients, CAG is recommended within $24 \mathrm{~h}$ in acute coronary syndrome patients without STE with a GRACE score $>140$ [27]. Rab et al. proposed an algorithm for the risk stratification of comatose CA patients. Time from collapse to ROSC of $30 \mathrm{~min}$ or more, arterial $\mathrm{pH}<7.2$, and lactate level $>7 \mathrm{mmol} / \mathrm{L}$ were included in the unfavorable resuscitation features. 
However, our results presented in Table 4 show that the benefit of early CAG was greater in high-risk patients. In contrast, low-risk patients did not significantly benefit from early CAG. Our research does not contradict the algorithm from Rab et al.; however, an improvement in cardiovascular SOFA scores by emergency CAG was found for high-risk patients, suggesting the possibility of improving their neurological prognosis.

The COACT trial, which is a recently published randomized controlled trial (RCT) for OHCA patients without STE, showed no significant differences in survival at 90 days between immediate CAG and delayed CAG [20]. A possible explanation for the difference between the COACT trial and our study might be the baseline characteristics of the enrolled patients. The COACT trial only included patients with shockable rhythm, which made up half of the patients in our study. Patients who received immediate CAG in the COACT trial had a shorter down time (15 min vs. $32 \mathrm{~min}$ ), higher levels of arterial $\mathrm{pH}$ (7.2 vs. 7.13), and lower levels of lactate on admission $(4.9 \mathrm{mmol} / \mathrm{L}$ vs. $9.15 \mathrm{mmol} / \mathrm{L})$ than those of the patients in our study. High-risk patients were more likely to be included in our study than in the COACT trial. Therefore, our study can provide additional information on patients not included in the COACT trial. Although observational studies may require additional RCTs and large-scale studies, our results suggest that early CAG should be performed in high-risk groups.

There were several limitations in this study. Our study is an observational prospective study and may lead to the risk of selection bias and residual confounding. An important possible explanation for the difference between the COACT trial and our study may be due to selection bias. Although the baseline characteristics, as well as the GRACE score, were not significantly different between the early CAG group and the no early CAG group in our study, the decision-making process of early CAG for each institution was inconsistent, and the possibility of CAG being performed earlier in patients with a predicted good prognosis cannot be excluded. CAG was not performed in $76.7 \%$ of the no early CAG group, which may have affected the outcome. However, the proportion of patients with PCI among the patients who underwent CAG was similar in both groups.

\section{Conclusions}

In conclusion, CAG performed within $24 \mathrm{~h}$ is associated with good neurologic outcome at 6 months after CA in patients without STE on ECG with presumed cardiac etiology treated with TTM. In particular, early CAG may be beneficial for high-risk patients (i.e., GRACE score $>140$, time from collapse to ROSC $>30$ min, cardiac enzyme elevation, arterial $\mathrm{pH}<7.2$, lactate $>7.0 \mathrm{mmol} / \mathrm{L}$, cardiac enzyme elevation and ST deviation).

Author Contributions: H.S., K.N.P. and C.S.Y. conceived and designed this study, interpreted the data and drafted the manuscript. H.J.K. and S.H.K. contributed to the acquisition of data and conducted data cleaning. W.Y.K., B.K.L., I.S.C., J.H.L. and C.S.Y. analyzed the data and interpreted the data. All authors have read and agreed to the published version of the manuscript.

Funding: This research received no external funding.

Institutional Review Board Statement: The study included an informed consent form approved by all participating hospitals, including the institutional review board (IRB) of Seoul St. Mary's Hospital (XC15OIMI0081K), and was registered at the International Clinical Trials Registry Platform (NCT02827422). Written informed consent was obtained from all patients' legal surrogates.

Informed Consent Statement: Not applicable.

Data Availability Statement: Available upon request.

Acknowledgments: The following investigators participated in the Korean Hypothermia Network. Network chair: Seung Pill Choi (The Catholic University of Korea, Eunpyeong St. Mary's Hospital); principal investigators of each hospital: Kyu Nam Park (The Catholic University of Korea, Seoul St. Mary's Hospital), Minjung Kathy Chae (Ajou University Medical Center), Won Young Kim (Asan Medical Center), Byung Kook Lee (Chonnam National University Hospital), Dong Hoon Lee (ChungAng University Hospital), Tae Chang Jang (Daegu Catholic University Medical Center), Jae Hoon 
Lee (Dong-A University Hospital), Yoon Hee Choi (Ewha Womans University Mokdong Hospital), Je Sung You (Gangnam Severance Hospital), Young Hwan Lee (Hallym University Sacred Heart Hospital), In Soo Cho (Hanil General Hospital), Su Jin Kim (Korea University Anam Hospital), JongSeok Lee (Kyung Hee University Medical Center), Yong Hwan Kim (Samsung Changwon Hospital), Min Seob Sim (Samsung Medical Center), Jonghwan Shin (Seoul Metropolitan Government Seoul National University Boramae Medical Center), Yoo Seok Park (Severance Hospital), Hyung Jun Moon (Soonchunhyang University Hospital Cheonan), Won Jung Jeong (The Catholic University of Korea, St. Vincent's Hospital), Joo Suk Oh (The Catholic University of Korea, Uijeongbu St. Mary's Hospital), Seung Pill Choi (The Catholic University of Korea, Yeouido St. Mary's Hospital), Kyoung-Chul Cha (Wonju Severance Christian Hospital).

Conflicts of Interest: None of the authors have declared a conflict of interest.

$\begin{array}{ll}\text { Abbreviations } & \\ \text { Out-of-hospital cardiac arrest } & \text { OHCA } \\ \text { Return of spontaneous circulation } & \text { ROSC } \\ \text { Targeted temperature management } & \text { TTM } \\ \text { Global Registry of Acute Coronary Events } & \text { GRACE } \\ \text { Korean Hypothermia Network } & \text { KORHN } \\ \text { Cerebrovascular accident } & \text { CVA } \\ \text { Transient ischemic attack } & \text { TIA }\end{array}$

\section{References}

1. Sandroni, C.; D'Arrigo, S.S.; Callaway, C.C.; Cariou, A.A.; Dragancea, I.I.; Taccone, F.S.; Antonelli, M. The rate of brain death and organ donation in patients resuscitated from cardiac arrest: A systematic review and meta-analysis. Intensive Care Med. 2016, 42, 1661-1671. [CrossRef] [PubMed]

2. Sasson, C.; Rogers, M.A.; Dahl, J.; Kellermann, A.L. Predictors of survival from out-of-hospital cardiac arrest: A systematic review and meta-analysis. Circ. Cardiovasc. Qual. Outcomes 2010, 3, 63-81. [CrossRef] [PubMed]

3. Kim, Y.J.; Kim, Y.H.; Lee, B.K.; Park, Y.S.; Sim, M.S.; Kim, S.J.; Oh, S.H.; Lee, D.H.; Kim, W.Y. Immediate versus early coronary angiography with targeted temperature management in out-of-hospital cardiac arrest survivors without ST-segment elevation: A propensity score-matched analysis from a multicenter registry. Resuscitation 2019, 135, 30-36. [CrossRef] [PubMed]

4. Spaulding, C.M.; Joly, L.-M.; Rosenberg, A.; Monchi, M.; Weber, S.N.; Dhainaut, J.-F.A.; Carli, P. Immediate coronary angiography in survivors of out-of-hospital cardiac arrest. N. Engl. J. Med. 1997, 336, 1629-1633. [CrossRef]

5. Dumas, F.; Cariou, A.; Manzo-Silberman, S.; Grimaldi, D.; Vivien, B.; Rosencher, J.; Empana, J.P.; Carli, P.; Mira, J.P.; Jouven, X.; et al. Immediate percutaneous coronary intervention is associated with better survival after out-of-hospital cardiac arrest: Insights from the PROCAT (Parisian Region Out of hospital Cardiac ArresT) registry. Circ. Cardiovasc. Interv. 2010, 3, $200-2007$. [CrossRef]

6. Callaway, C.W.; Donnino, M.W.; Fink, E.L.; Geocadin, R.G.; Golan, E.; Kern, K.B.; Leary, M.; Meurer, W.J.; Peberdy, M.A.; Thompson, T.M.; et al. Part 8: Post-Cardiac Arrest Care: 2015 American Heart Association Guidelines Update for Cardiopulmonary Resuscitation and Emergency Cardiovascular Care. Circulation 2015, 132, S465-S482. [CrossRef]

7. Monsieurs, K.G.; Nolan, J.P.; Bossaert, L.L.; Greif, R.; Maconochie, I.K.; Nikolaou, N.I.; Perkins, G.D.; Soar, J.; Truhlár̆, A.; Wyllie, J.; et al. European Resuscitation Council Guidelines for Resuscitation 2015: Section 1. Executive summary. Resuscitation 2015, 95, 1-80. [CrossRef]

8. Kern, K.B.; Lotun, K.; Patel, N.; Mooney, M.R.; Hollenbeck, R.D.; McPherson, J.A.; McMullan, P.W.; Unger, B.; Hsu, C.H.; Seder, D.B.; et al. Outcomes of Comatose Cardiac Arrest Survivors with and Without ST-Segment Elevation Myocardial Infarction: Importance of Coronary Angiography. JACC Cardiovasc. Interv. 2015, 8, 1031-1040. [CrossRef]

9. Dankiewicz, J.; Nielsen, N.; Annborn, M.; Cronberg, T.; Erlinge, D.; Gasche, Y.; Hassager, C.; Kjaergaard, J.; Pellis, T.; Friberg, H. Survival in patients without acute ST elevation after cardiac arrest and association with early coronary angiography: A post hoc analysis from the TTM trial. Intensive Care Med. 2015, 41, 856-864. [CrossRef]

10. Millin, M.G.; Comer, A.C.; Nable, J.V.; Johnston, P.V.; Lawner, B.J.; Woltman, N.; Levya, M.J.; Seamane, K.G.; Hirshond, J.M. Patients without ST elevation after return of spontaneous circulation may benefit from emergent percutaneous intervention: A systematic review and meta-analysis. Resuscitation 2016, 108, 54-60. [CrossRef]

11. Elfwén, L.; Lagedal, R.; James, S.; Jonsson, M.; Jensen, U.; Ringh, M.; Claesson, A.; Oldgren, J.; Herlitz, J.; Rubertsson, S.; et al. Coronary angiography in out-of-hospital cardiac arrest without ST elevation on ECG-Short- and long-term survival. Am. Heart. J. 2018, 200, 90-95. [CrossRef] [PubMed]

12. Doorey, A.; Garratt, K.N.; Weintraub, W.W. Coronary Angiography After Out-of-Hospital Cardiac Arrest. JACC Cardiovasc. Interv. 2017, 10, 963. [CrossRef] [PubMed] 
13. Radsel, P.; Noc, M. Immediate coronary angiography after cardiac arrest-Friend or foe? Resuscitation 2018, 132, A5-A6. [CrossRef] [PubMed]

14. Cigarroa, J.E. Out-of-Hospital Cardiac Arrest Survivors in Patients Without ST-Segment Elevation Infarction: Is Routine Coronary Angiography Reasonable? JACC Cardiovasc. Interv. 2016, 9, 1019-1021. [CrossRef]

15. Kim, Y.J.; Min, S.-Y.; Lee, D.H.; Lee, B.K.; Jeung, K.W.; Lee, H.J.; Shin, J.; Ko, B.S.; Ahn, S.; Nam, G.-B.; et al. The Role of Post-Resuscitation Electrocardiogram in Patients with ST-Segment Changes in the Immediate Post-Cardiac Arrest Period. JACC. Cardiovasc. Interv. 2017, 10, 451-459. [CrossRef]

16. Bro-Jeppesen, J.; Kjaergaard, J.; Wanscher, M.; Pedersen, F.; Holmvang, L.; Lippert, F.K.; Møller, J.E.; Køber, L.; Hassager, C. Emergency coronary angiography in comatose cardiac arrest patients: Do real-life experiences support the guidelines? Eur. Hear. J. Acute Cardiovasc. Care 2012, 1, 291-301. [CrossRef]

17. Dumas, F.; Bougouin, W.; Geri, G.; Lamhaut, L.; Rosencher, J.; Pène, F.; Chiche, J.-D.; Varenne, O.; Carli, P.; Jouven, X.; et al. Emergency Percutaneous Coronary Intervention in Post-Cardiac Arrest Patients Without ST-Segment Elevation Pattern: Insights from the PROCAT II Registry. JACC Cardiovasc. Interv. 2016, 9, 1011-1018. [CrossRef]

18. Hollenbeck, R.D.; McPherson, J.A.; Mooney, M.R.; Unger, B.T.; Patel, N.C.; McMullan, P.W.; Hsu, C.-H.; Seder, D.B.; Kern, K.B. Early cardiac catheterization is associated with improved survival in comatose survivors of cardiac arrest without STEMI. Resuscitation 2014, 85, 88-95. [CrossRef]

19. Staudacher, I.I.; den Uil, C.; Jewbali, L.; van Zandvoort, L.; Zijlstra, F.; Van Mieghem, N.; Van Mieghem, N.; Boersma, E.; Daemen, J. Timing of coronary angiography in survivors of out-of-hospital cardiac arrest without obvious extracardiac causes. Resuscitation 2018, 123, 98-104. [CrossRef]

20. Lemkes, J.S.; Janssens, G.N.; Van Der Hoeven, N.W.; Jewbali, L.S.; Dubois, E.A.; Meuwissen, M.; Rijpstra, T.A.; Bosker, H.A.; Blans, M.J.; Bleeker, G.B.; et al. Coronary Angiography after Cardiac Arrest without ST-Segment Elevation. N. Engl. J. Med. 2019, 380, 1397-1407. [CrossRef]

21. Nikolaou, N.I.; Welsford, M.; Beygui, F.; Bossaert, L.; Ghaemmaghami, C.; Nonogi, H.; O'Connor, R.E.; Pichel, D.R.; Scott, T.; Walters, D.L.; et al. Part 5: Acute coronary syndromes: 2015 International Consensus on Cardiopulmonary Resuscitation and Emergency Cardiovascular Care Science with Treatment Recommendations. Resuscitation 2015, 95, e121-e146. [CrossRef] [PubMed]

22. Rab, T.; Kern, K.B.; Tamis-Holland, J.E.; Henry, T.D.; McDaniel, M.; Dickert, N.W.; Cigarroa, J.E.; Keadey, M.; Ramee, S. Interventional Council, American College of Cardiology. Cardiac Arrest: A Treatment Algorithm for Emergent Invasive Cardiac Procedures in the Resuscitated Comatose Patient. J. Am. Coll. Cardiol. 2015, 66, 62-73. [CrossRef] [PubMed]

23. Amsterdam, E.A.; Wenger, N.K.; Brindis, R.G.; Casey, D.E., Jr.; Ganiats, T.G.; Holmes, D.R., Jr.; Jaffe, A.S.; Jneid, H.; Kelly, R.F.; Kontos, M.C.; et al. 2014 AHA/ACC guideline for the management of patients with non-ST-elevation acute coronary syndromes: Executive summary: A report of the American College of Cardiology/American Heart Association Task Force on Practice Guidelines. Circulation 2014, 130, 2354-2394. [CrossRef] [PubMed]

24. Deharo, P.; Ducrocq, G.; Bode, C.; Cohen, M.; Cuisset, T.; Mehta, S.R.; Pollack, C., Jr.; Wiviott, S.D.; Elbez, Y.; Sabatine, M.S.; et al. Timing of Angiography and Outcomes in High-Risk Patients with Non-ST-Segment-Elevation Myocardial Infarction Managed Invasively: Insights From the TAO Trial (Treatment of Acute Coronary Syndrome With Otamixaban). Circulation 2017, 136, 1895-1907. [CrossRef] [PubMed]

25. Tsai, M.S.; Sung, C.W.; Chen, W.J.; Chuang, P.Y.; Wang, C.H.; Wu, Y.W.; Chang, W.-Y.; Chen, W.T.; Huanga, C.-H. Stenosis and revascularization of the coronary artery are associated with outcomes in presumed cardiogenic arrest survivors: A multi-center retrospective cohort study. Resuscitation 2019, 137, 52-60. [CrossRef] [PubMed]

26. Khan, M.S.; Shah, S.M.M.; Mubashir, A.; Khan, A.R.; Fatima, K.; Schenone, A.L.; Khosa, F.; Samady, H.; Menon, V. Early coronary angiography in patients resuscitated from out of hospital cardiac arrest without ST-segment elevation: A systematic review and meta-analysis. Resuscitation 2017, 121, 127-134. [CrossRef]

27. Elbarouni, B.; Goodman, S.G.; Yan, R.T.; Welsh, R.C.; Kornder, J.M.; Deyoung, J.P.; Wong, G.C.; Rose, B.; Grondin, F.R.; Gallo, R.; et al. Validation of the Global Registry of Acute Coronary Event (GRACE) risk score for in-hospital mortality in patients with acute coronary syndrome in Canada. Am. Hear. J. 2009, 158, 392-399. [CrossRef] 